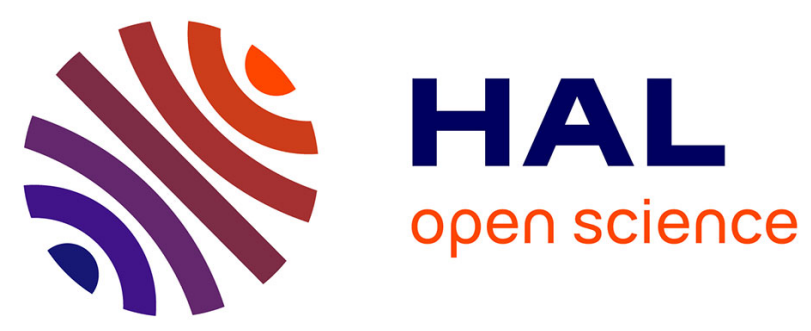

\title{
Variability in Dispersal Syndromes Is a Key Driver of Metapopulation Dynamics in Experimental Microcosms
}

Staffan Jacob, Alexis Chaine, Michèle Huet, Jean Clobert, Delphine Legrand

\section{To cite this version:}

Staffan Jacob, Alexis Chaine, Michèle Huet, Jean Clobert, Delphine Legrand. Variability in Dispersal Syndromes Is a Key Driver of Metapopulation Dynamics in Experimental Microcosms. American Naturalist, 2019, 194 (5), pp.613-626. 10.1086/705410 . hal-02347855

\section{HAL Id: hal-02347855 \\ https://cnrs.hal.science/hal-02347855}

Submitted on 26 Nov 2020

HAL is a multi-disciplinary open access archive for the deposit and dissemination of scientific research documents, whether they are published or not. The documents may come from teaching and research institutions in France or abroad, or from public or private research centers.
L'archive ouverte pluridisciplinaire HAL, est destinée au dépôt et à la diffusion de documents scientifiques de niveau recherche, publiés ou non, émanant des établissements d'enseignement et de recherche français ou étrangers, des laboratoires publics ou privés. 


\title{
Variability in Dispersal Syndromes Is a Key Driver of Metapopulation Dynamics in Experimental Microcosms
}

\author{
Staffan Jacob, ${ }^{1,2, \star}$ Alexis S. Chaine, ${ }^{1,3}$ Michèle Huet, ${ }^{1}$ Jean Clobert, ${ }^{1}$ and Delphine Legrand ${ }^{1}$ \\ 1. Evolutionary Ecology Group, Station d'Ecologie Théorique et Expérimentale du CNRS UMR5321, 2 route du CNRS, 09200 Moulis, \\ France; 2. Biodiversity Research Centre, Earth and Life Institute, Université Catholique de Louvain, Croix du Sud 4, L7-07-04, \\ 1348 Louvain-la-Neuve, Belgium; 3. Institute for Advanced Studies in Toulouse, Toulouse School of Economics, \\ 21 allée de Brienne, 31015 Toulouse, France
}

Submitted June 27, 2018; Accepted April 26, 2019; Electronically published September 13, 2019

Online enhancements: supplemental figures and tables. Dryad data: https://doi.org/10.5061/dryad.r63jf31.

ABSTRACT: Evolutionary ecology studies have increasingly focused on the impact of intraspecific variability on population processes. However, the role such variation plays in the dynamics of spatially structured populations and how it interacts with environmental changes remains unclear. Here we experimentally quantify the relative importance of intraspecific variability in dispersal-related traits and spatiotemporal variability of environmental conditions for the dynamics of two-patch metapopulations using clonal genotypes of a ciliate in connected microcosms. We demonstrate that in our simple two-patch microcosms, differences among genotypes are at least as important as spatiotemporal variability of resources for metapopulation dynamics. Furthermore, we show that an important proportion of this effect results from variability of dispersal syndromes. These syndromes can therefore be as important for metapopulation dynamics as spatiotemporal variability of environmental conditions. This study demonstrates that intraspecific variability in dispersal syndromes can be key in the functioning of metapopulations facing environmental changes.

Keywords: intraspecific variability, dispersal syndrome, eco-evolutionary dynamics, spatially structured populations, genetic diversity, environmental changes.

\section{Introduction}

Organisms live in a spatially and temporally structured world, meaning that populations are distributed in spatially structured habitats whose suitability changes through time (Hanski and Gaggiotti 2004). Since the environmental conditions encountered in a habitat govern an organisms' reproductive success and survival, the dynamics of local populations are affected by the extent of spatiotemporal variability in environmental conditions (Moran 1953; Levin 1976; Bascompte and Solé 1998; Hanski 1998). Such spatiotempo-

\footnotetext{
* Corresponding author; email: jacobstaffan@gmail.com. ORCIDs: Jacob, https://orcid.org/0000-0003-1956-9646.

Am. Nat. 2019. Vol. 194, pp. 000-000. (C) 2019 by The University of Chicago. 0003-0147/2019/19405-58577\$15.00. All rights reserved. DOI: $10.1086 / 705410$
}

rally variable selective pressures can drive changes in the distribution of phenotypic traits in populations (Kettlewell 1973; Endler 1980; Stearns 1983). Beyond being the target of selection, phenotypic traits can in turn influence ecological dynamics, such as population demography and community composition (Pimentel 1968; Thompson 1998; Urban et al. 2008; Pelletier et al. 2009; Hendry 2017; Legrand et al. 2017). Furthermore, although variability among species is of considerable importance, intraspecific variability can have an equivalent or even sometimes greater impact on ecological processes than interspecific variability (Des Roches et al. 2018; Raffard et al. 2019). Estimating the importance of genetic and phenotypic intraspecific variability relative to environmental variability for the dynamics of spatially structured populations is therefore one of the core current challenges in ecological and evolutionary research (Bolnick et al. 2003, 2011; Violle et al. 2012; Des Roches et al. 2018).

Dispersal is recognized as one of the central traits driving the dynamics of spatially structured populations (MacArthur and Wilson 1963; Hanski 1998; Lenormand 2002; Bowler and Benton 2005; Ronce 2007; Abbott 2011; Clobert et al. 2012). Since the establishment of metapopulation theory, a large body of theoretical studies has been dedicated to understanding and predicting the dynamics and persistence of spatially structured populations and the evolution of dispersal in fragmented landscapes (reviewed in Clobert et al. 2009, 2012; Duputié and Massol 2013). However, most of the theoretical framework on this topic assumes dispersal to be random with regard to the phenotype or the environmental context, and these simplifying assumptions contrast with increasing evidence from empirical studies for extensive variability in phenotypic and behavioral traits related to dispersal movements (reviewed in Bowler and Benton 2005; Ronce 2007; Clobert et al. 2012; Travis et al. 2012; Jacob et al. 2015a; Cote et al. 2017). Indeed, dispersal often depends on a variety of internal factors enabling or enhancing its 
success (e.g., orientation skills, energy reserves, fecundity, niche breadth) and external factors affecting individual fitness (e.g., population density, resources, temperature; Clobert et al. 2009; Stevens et al. 2014; Legrand et al. 2015; Cote et al. 2017). Multiple dispersal-related traits are most often assembled in dispersal syndromes, defined as the covariation between dispersal and other phenotypic traits (Fjerdingstad et al. 2007; Clobert et al. 2009; Stevens et al. 2014; Cote et al. 2017).

The existence of great variability in dispersal-related traits and strategies within species is a challenge for our ability to forecast ecological and evolutionary dynamics under environmental variability (Clobert et al. 2009). This is because the role of dispersal in the persistence of spatially structured populations may drastically differ depending on the dispersalrelated traits involved and the environmental conditions encountered (reviewed in Bowler and Benton 2005; Clobert et al. 2009; Jacob et al. 2015a). For instance, theoretical models revealed that intraspecific variation, including colonizing strategies, might increase metapopulation persistence (Leimar and Norberg 1997). Furthermore, compared with random dispersal, phenotype-dependent dispersal decisions can favor local adaptation (Holt and Barfield 2015; Jacob et al. 2017), stabilize metapopulation dynamics (Mortier et al. 2019), or mediate specialist-generalist coexistence (Jacob et al. 2018). However, models also revealed that phenotypic variability can have either positive or negative effects on metapopulation stability depending on the mismatch between dispersal phenotypes and local conditions (Gibert 2016). Besides, the success of these different dispersal strategies should differ depending on the environmental context faced. Unfortunately, few theoretical predictions are available on this topic, because most models usually consider dispersal to be a random process (e.g., Wang et al. 2015). One notable exception for the interaction between dispersal strategies and environmental variability on metapopulation dynamics lies in a model from Rodrigues and Johnstone (2014), who showed that spatiotemporal variability of environmental conditions should favor positive density-dependent dispersal, while spatially variable but temporally stable environments should favor negative density-dependent dispersal.

However, experimental approaches quantifying the importance of different dispersal-related traits for the dynamics of spatially structured populations are still crucially lacking, especially under varying environmental conditions, as has been pointed out repeatedly (e.g., Travis et al. 2012; Jacob et al. 2015a; Cote et al. 2017; Hendry 2017; Legrand et al. 2017). This experimental gap mostly results from the constraints in handling the required replicated experiments of spatially structured populations followed over sufficient temporal scales. Experimental micro/mesocosms using shortgeneration-time species provide a valuable opportunity to fill this gap (Gause 1934; Jessup et al. 2004; Legrand et al.
2012; Altermatt et al. 2015; De Roissart et al. 2015), offering especially the possibility to quantify the importance of dispersal strategies for the dynamics of metapopulations facing different regimes of environmental variability.

Here we experimentally quantified the relative effects of intraspecific variability in dispersal-related traits and environmental variability for the dynamics of simple experimental metapopulations. Importantly, we aimed at expanding our understanding of the impact of dispersal syndromes on the dynamics of spatially structured populations. We thus did not compare the dynamics of metapopulations composed of different levels of intraspecific variability but separately quantified the effects of distinct genotypes and phenotypes for metapopulation dynamics. To do so, we used the ciliated protist Tetrahymena thermophila in simplified metapopulations consisting of replicated two-patch microcosms. We first characterized dispersal strategies and related phenotypic traits among six isogenic strains (hereafter called "genotypes"), including dispersal propensity, resource- and density-dependent dispersal, phenotypic specialization, and growth rate (fig. 1). Second, these characterized genotypes were separately introduced in experimental two-patch microcosms exposed to different regimes of spatial and temporal variability of resources and followed over 5 weeks ( 200 generations). First, we expected that metapopulation dynamics would differ as a result of intraspecific variation in dispersalrelated traits (Leimar and Norberg 1997; Gibert 2016). We also predicted that genotypes with traits enhancing dispersal would generate more homogeneous metapopulations than genotypes with reduced dispersal ability (Clobert et al. 2009; Cote et al. 2017). Second, we expected environmental variability to affect metapopulation dynamics as a result of differences in patch quality and therefore population growth. Temporal variability in environmental conditions, through its effect on population growth, should increase the temporal variability of metapopulation size compared with stable environmental conditions. In contrast, metapopulations should show higher spatial variability in local population sizes under spatially variable environmental conditions. Finally, we expected the dynamics of these simple experimental metapopulations to vary depending on the interaction between the characteristics of genotypes and the environmental context. In particular, specialized dispersal strategies (e.g., high dispersal rate, morphological specialization for dispersal) might buffer the effects of environmental variability on metapopulation dynamics.

\section{Methods \\ Study Organism}

The study organism Tetrahymena thermophila is a freshwater ciliate commonly used in experimental ecology and 

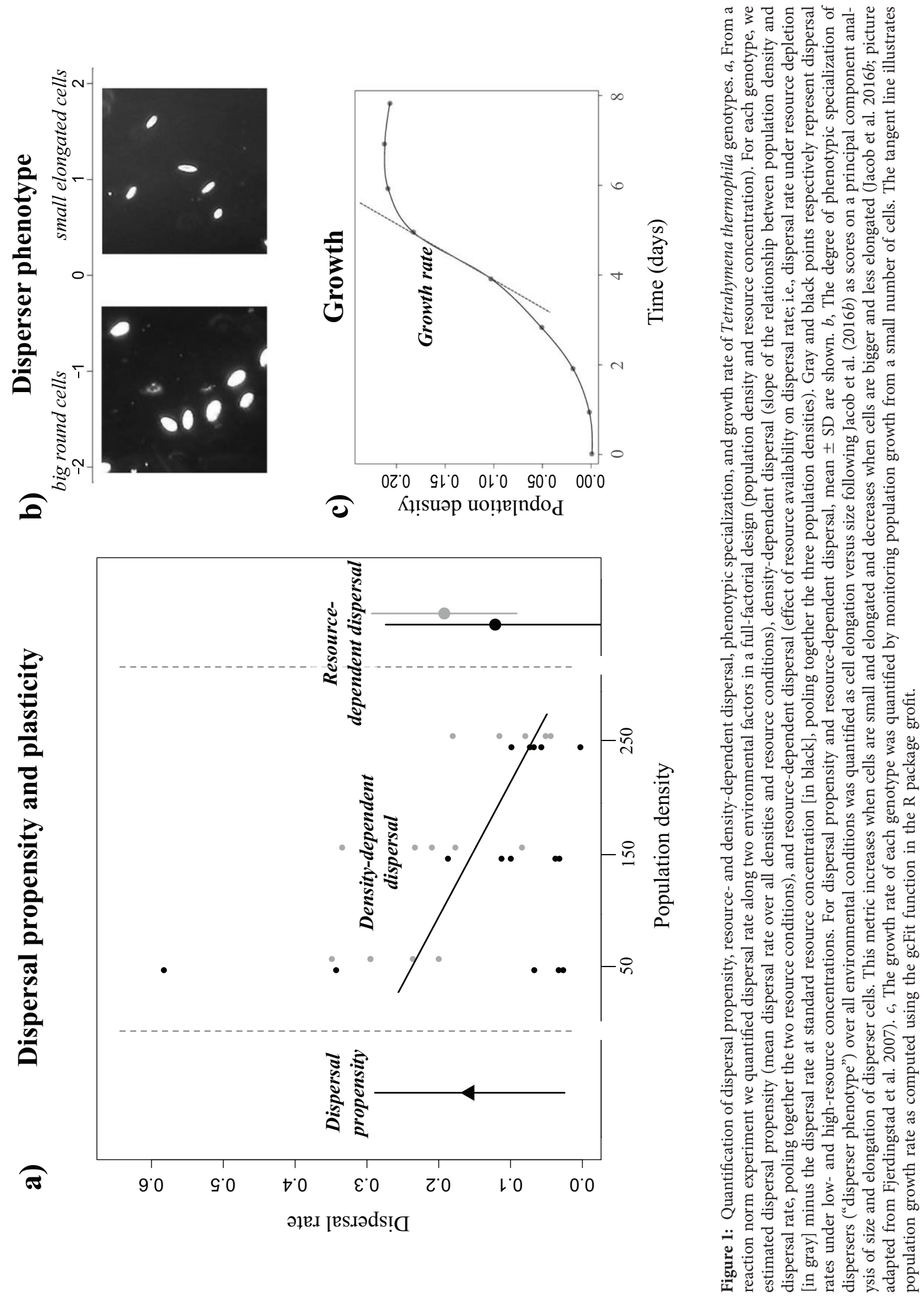

This content downloaded from 129.125.019.061 on October 03, 2019 23:52:22 PM 
evolution (Pennekamp et al. 2014; Altermatt et al. 2015; Fronhofer and Altermatt 2015; Jacob et al. 2015b, 2016a, $2016 b, 2017)$. Clonally reproducing isogenic strains are typically maintained at $23^{\circ} \mathrm{C}$ in synthetic liquid growth medium ( $2 \%$ Difco proteose peptone, $0.2 \%$ yeast extract) and propagated once a week by transferring $10 \mu \mathrm{L}$ (i.e., $~ 100$ cells) into fresh medium. Here we used six genotypes originally sampled in North America by P. Doerder that are available at the Tetrahymena Stock Center (table S1; tables S1-S3 are available online; see Pennekamp et al. 2014; Jacob et al. 2017).

\section{Experiments}

The experimental procedure consisted of two steps, as described below. First, we quantified dispersal-related phenotypic variability among six genotypes of $T$. thermophila. Second, we submitted those characterized genotypes to four environmental variability regimes in two-patch microcosms, allowing us to quantify the relative importance of amonggenotype differences and environmental variability for the dynamics of simple experimental metapopulations.

Experiment 1: Quantification of Genotype-Specific Dispersal Strategies and Related Phenotypic Traits. Our available stock of isogenic lines strongly differ in their phenotypic and lifehistory traits as previously measured in standard resource conditions (Fjerdingstad et al. 2007; Pennekamp et al. 2014; Jacob et al. 2015b, 2016a, 2016b, 2018). We extended this characterization by quantifying dispersal decisions and related phenotypic traits using a standard reaction norm approach on six genotypes (table S1; see Pennekamp et al. 2014; Jacob et al. 2017). We used connected microcosms consisting of two habitat patches (1.5-mL microtubes) connected by a corridor (silicone tube, $4 \mathrm{~mm}$ internal diameter, $2.5 \mathrm{~cm}$ long, meaning $10 \%$ of the total volume of the two-patch microcosms; Chaine et al. 2010; Pennekamp et al. 2014; Jacob et al. 2015b, 2016b). We quantified dispersal strategies along two environmental factors in a replicated full-factorial design: population density (three levels: 50,000, 150,000 and 250,000 cells/mL; see Pennekamp et al. 2014; Jacob et al. $2016 b$ ) and habitat quality (two conditions: a standard concentration and a low-resource treatment with nine times fewer resources, resulting in growth rates $22.68 \pm 10.83$ (mean \pm $\mathrm{SE})$ times lower compared with the standard condition).

Both this initial quantification of dispersal strategies and the metapopulation dynamics experiment (see below) were performed using an agar-based nutrient system. Different concentrations of resources were initially placed in $100 \mu \mathrm{L}$ of $1.5 \%$ agar at the bottom of the microtubes. Once the agar solutions solidified, tubes were filled with water. This method limits the homogenization of nutrients between patches observed in water-based nutrient solutions and thus allowed us to generate spatial heterogeneity of resources that is still visible after 4 days (nutrients are naturally colored).

To quantify dispersal strategies, five replicates of each genotype were placed in the "start patch" of standard twopatch systems for each combination of cell density and resource treatments (three densities $\times$ two levels of resources; see above), while the other patch (hereafter called the "target patch") was initially free of cells and filled with standard resource concentration. Dispersal was allowed for $5 \mathrm{~h}$ (Pennekamp et al. 2014; Jacob et al. 2016a), and we used automatic analysis of digital pictures taken under dark-field microscopy (Axio Zoom V16; Zeiss) to quantify population sizes and cell morphology in the start (residents) and target (dispersers) patches (Pennekamp and Schtickzelle 2013; Pennekamp et al. 2014; Jacob et al. 2015b, 2016b). As illustrated in figure 1 , for each genotype we quantified dispersal propensity, density-dependent dispersal, resource-dependent dispersal, disperser phenotype, and growth rate. Dispersal propensity is defined as the mean dispersal rate over all environmental conditions (i.e., three densities $\times$ two resources conditions), indicative of the genotype's general tendency to disperse. Density-dependent dispersal is the slope of the relationship between population density and dispersal rate, pooling together the two resource concentrations, meaning, for instance, that negative values denote a tendency to preferentially leave low-density patches. Resource-dependent dispersal is the effect of resource availability on dispersal rate, quantified as the dispersal rate under resource depletion minus the dispersal rate at standard resource concentration (pooling the three population densities). Genotypes increasing their dispersal rate when resources are depleted are characterized by positive values of resource-dependent dispersal, while those decreasing dispersal rate when resources are depleted show negative resource-dependent dispersal. Disperser phenotype is cell elongation versus size following Jacob et al. (2016b), as scores of a principal component analysis (PCA) on size and elongation of disperser cells. This metric increases when cells are small and elongated and decreases when cells are bigger and less elongated (Jacob et al. 2016b). We calculated this metric because in T. thermophila, small and elongated cells are usually specialized for long-distance movements and show greater swim speed and straighter movements (Nelsen 1978; Fjerdingstad et al. 2007; Schtickzelle et al. 2009; Pennekamp et al. 2014; Jacob et al. 2016b). Finally, growth rate was quantified by monitoring population growth from a small number of cells in single unconnected microtubes (absorbance measurements at $550 \mathrm{~nm}$ every day for 1 week growth rate computed using the gcFit function in the R package grofit). Preliminary tests revealed that growth rate differed among genotypes $\left(F_{5,42}=7.59, P<.001\right)$ and along resource concentration $\left(F_{1,42}=5.65, P=.02\right)$ but that genotypes did not significantly differ in their response to 
resource concentration (genotype $\times$ resource interaction: $\left.F_{5,42}=0.17, P=.97\right)$. We therefore used growth at standard resource concentration as our measure of genotypes' growth rate in all analyses.

Experiment 2: Metapopulation Dynamics under Environmental Variability. The experimental system of simplified metapopulations consisted of the same two-patch microcosms as used in experiment 1 (see above). For each of the six genotypes and four environmental variability regimes we constructed five experimental systems, resulting in a total of 120 two-patch microcosms (six genotypes $\times$ four environmental variability regimes $\times$ five replicates; initial density, 50,000 cells $/ \mathrm{mL}$ ). Twice a week for 5 weeks ( 200 generations) the size of local populations was quantified through picture analysis, and $10 \%$ of each local population was transferred into the corresponding patch of a new two-patch microcosm with fresh agar-based nutrients (see above). The spatial/temporal variability of resources was manipulated under four environmental variability regimes, which were set up in order to maintain equal quantities of resources among treatments at the two-patch microcosm level over time. The homogeneous regime consisted of metapopulations with half of standard resource concentration in each patch. Temporal variability was set up with an alternation of depleted and standard resource concentration in the two patches twice a week (i.e., 20 generations), with the two patches containing equal resource conditions. Spatial variability consisted of one patch with low-resource concentration and the other at standard concentration, with fixed spatial distribution of resources over time. Finally, spatiotemporal variability was similar to spatial variability but inverting the patch resources twice a week, leading to regular and predictable spatiotemporal variability of resources.

From local population sizes estimated twice a week we computed three descriptors of the dynamics of these twopatch microcosms: metapopulation size (sum of local population sizes at each time), spatial variability in population size (coefficient of variation of local population sizes at each time; R packages cvar and BioStatR; Bertrand and MaumyBertrand 2010), and temporal variability in metapopulation size (coefficient of variation of metapopulation size through time).

Finally, we reiterated the quantification of dispersal traits at the end of the experiment to test for evolution of genotypes' traits. As we found no effect of environmental variability regimes on the dispersal traits of genotypes after 200 generations in the two-patch microcosms (table S2), we used initial phenotypic values in all analyses.

Statistical Analyses. The architecture of genotypes' traits measured in experiment 1 was explored using Pearson correlation coefficients, and their organization in dispersal syndromes was quantified using PCA (dudi.pca function, $\mathrm{R}$ package ade4). The contribution of variables to each principal component (fig. 2) was determined by comparing the observed contributions to a uniform distribution using the fviz_contrib function (R package factoextra). Since growth rate is expected to be an important driver of ecological dynamics, we included it as a separate trait in the analyses (see below) and thus excluded it from the PCA of dispersalrelated traits.

We tested for the influence of genotype identity and environmental variability on metapopulation dynamics using linear models ( $\mathrm{lm}$ function, $\mathrm{R}$ package stats). Following De Roissart et al. (2015), we used the mean of metapopulation dynamic descriptors over time as dependent variables in models (i.e., metapopulation size and spatial variability in population size; temporal variability in metapopulation size being already computed over time; see above) over time as dependent variables in models, and explanatory factors were environmental variability treatments, genotype identity, and their interaction. Following a backward-selection procedure, we removed explanatory factors and their interaction from the models when nonsignificant at the $P \leq .05$ level. Conducting analyses separately through time step led to qualitatively similar conclusions (fig. S1, available online; table S3). To estimate the relative contribution of each explanatory factor to metapopulation dynamics, we conducted variance partitioning analyses using calc.relimp with the lmg metric (R package relaimpo; Lindeman et al. 1980; Groemping 2006).

Following the above-described analyses that aimed at quantifying the importance of intraspecific variability and environmental variability for metapopulation dynamics, we more specifically tested the importance of variability in dispersal-related traits among genotypes compared with environmental variability for ecological dynamics. To do so, we used linear models followed by variance partitioning, as described above, to test the effect of environmental variability treatments, dispersal syndromes (summarized in PCA axes; see fig. 2), genotype-specific growth rates, and their interaction for metapopulation size and variability. Quadratic effects were initially included but not presented in the table because they were not significant.

\section{Results \\ Architecture of Dispersal Strategies}

From the multiple traits we quantified in this study, we first examined the patterns of covariation between traits using Pearson correlations. We found that dispersal propensity was correlated to dispersal phenotype and resource-dependent dispersal (fig. 2a), meaning that the more dispersive genotypes showed specialized dispersal phenotypes and increased 


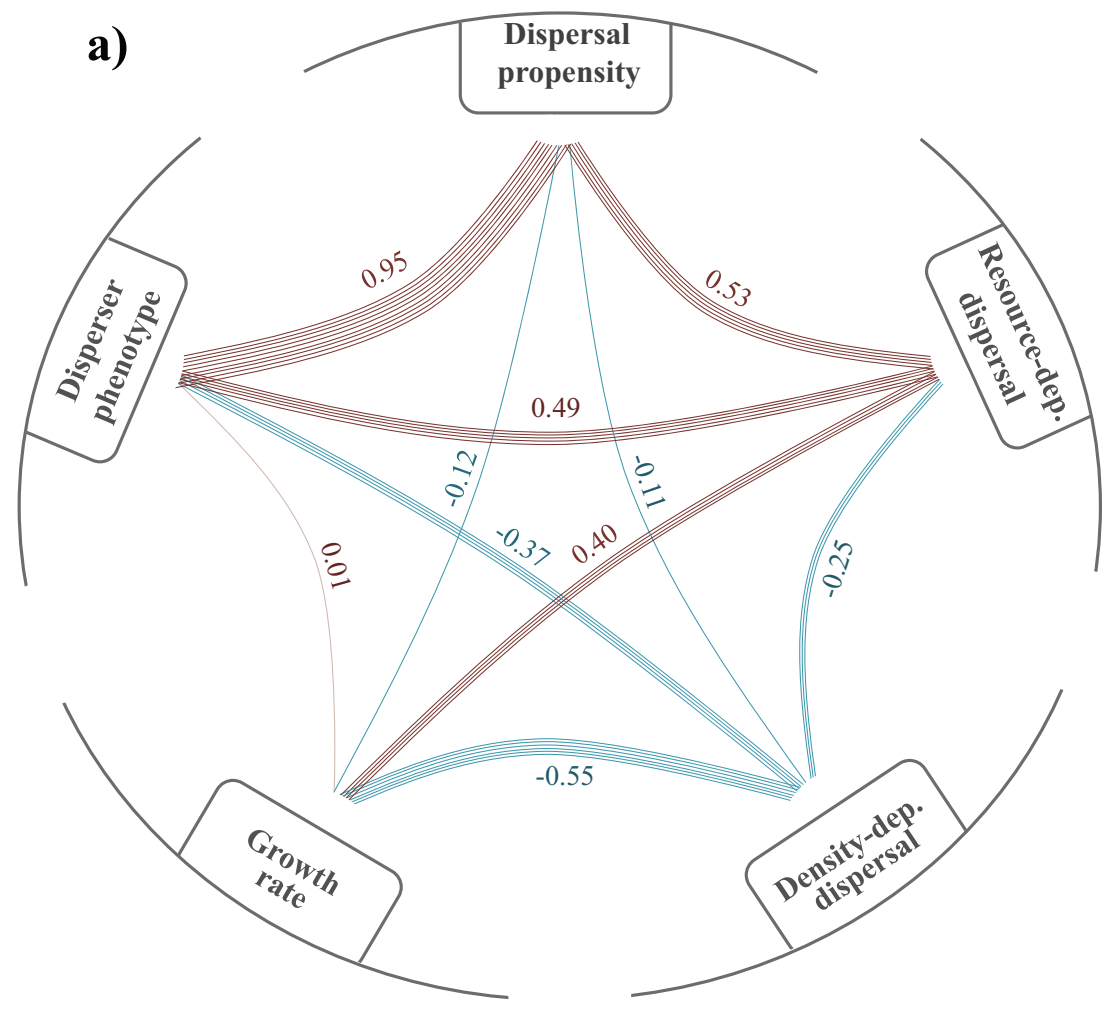

b) Components of dispersal syndromes

\begin{tabular}{lccc} 
& $\begin{array}{c}\text { PC 1 } \\
\text { Dispersal specialization } \\
\text { and resource-dependency }\end{array}$ & & $\begin{array}{c}\text { Pensity-dependent } \\
\text { dispersal }\end{array}$ \\
\cline { 2 - 2 } Dispersal propensity & $\mathbf{0 . 9 1}$ & 0.35 \\
Disperser phenotype & $\mathbf{0 . 9 5}$ & 0.08 \\
Resource-dependent dispersal & $\mathbf{0 . 7 3}$ & -0.01 \\
Density-dependent dispersal & -0.44 & $\mathbf{0 . 8 9}$
\end{tabular}

Figure 2: Architecture of traits in Tetrahymena thermophila in the six genotypes used in this study. $a$, Correlations among pairs of traits. Pearson correlation coefficients are shown, with the width of the lines connecting traits proportional to the strength of correlations (red for positive correlation, blue for negative correlations). $b$, Organization of dispersal-related traits in dispersal syndromes. The contributions of variables to each principal component (highlighted in bold) were defined compared with uniform distribution of contributions using the fviz_contrib function in the R package factoextra. The first axis comprises $61.4 \%$ of original variance, and the second $23.1 \%$. Since growth rate is expected to be an important driver of population dynamics, it was excluded from the principal component analysis and included as a separate trait in the analyses (see "Methods").

their tendency to disperse when local resources are depleted. On the other side, genotypes' growth rate and densitydependent dispersal were weakly correlated with the three other traits and correlated negatively with each other (fig. $2 a$ ). This means that genotypes with the highest growth rate exhibited negative density-dependent dispersal. Then we summarized the variance in these dispersal-related traits using
PCA, while excluding growth rate from the PCA to include it as a separate trait in the analyses (see "Methods"). The PCA provided evidence for two dimensions of dispersal syndromes (fig. $2 b ; 84.5 \%$ of total variance explained by the first two axes; $61.4 \%$ on the first axis and $23.1 \%$ on the second). The first axis comprised dispersal propensity, disperser phenotype, and resource-dependent dispersal, which generally 
describe a genotype's dispersal specialization and resource dependency (fig. 2b). When increasing values on this first axis, genotypes show higher dispersal propensity, display more specialized dispersal phenotype (small and elongated cells), and preferentially leave poor patches compared with rich ones. The second axis includes density-dependent dispersal, with higher axis values for genotypes that have a positive density-dependent dispersal (meaning that dispersal increases when density increases).

\section{Drivers of Metapopulation Dynamics}

As expected, environmental variability was an important driver of the dynamics of experimental two-patch microcosms (fig. 3; table 1, pt. A). Metapopulation size was on average the highest in a homogeneous environment, followed by spatiotemporally and spatially variable environments, and the lowest under temporally variable environments (fig. 3). Spatial variability in population sizes was higher under spatial and spatiotemporal environmental variability than under temporally variable and homogeneous environments (fig. 3). Finally, we found higher temporal variability in population size under temporal environmental variability compared with a homogeneous environment, with intermediate values under spatial and spatiotemporal environmental variability (fig. 3).

Although the environmental variability regimes were of great importance as developed above, their effects on the dynamics of experimental two-patch microcosms differed among genotypes. Furthermore, differences among genotypes were overall more important for these dynamics than environmental variability treatments in our experiment (table 1, pt. A). In particular, we found $73 \%$ of variance in metapopulation size explained by genotype and $4 \%$ by the interaction between environmental variability and genotype, while $19 \%$ was attributed to environmental variability (total variance explained, 96\%; table 1, pt. A). Regarding spatial variability of population size, environmental variability and genotype identity appeared to be of similar importance: $24 \%$ of spatial variability in population size was attributed to genotype, $34 \%$ to environmental variability, and $22 \%$ to the genotype $\times$ environmental variability interaction (total variance explained, $80 \%$; table 1, pt. A). Finally, $39 \%$ of temporal variability in metapopulation size was explained by environmental variability per se, and respectively $52 \%$ and $3 \%$ was attributed to genotype and the genotype $\times$ environmental variability interaction (total variance explained, 94\%; table 1, pt. A).

We then tested to what extent the effects of genotype on metapopulation dynamics result from variability in dispersalrelated traits. We found that dispersal-related traits (summarized in PCA axes as described above; see fig. 2) play an important role for the dynamics of two-patch microcosms, affecting metapopulation size and the spatial and temporal variability in metapopulation size (fig. 3; table 1, pt. B). Metapopulation size increased with dispersal specialization and resource dependency (PC1; 6\% of variance explained) and density-dependent dispersal (PC2; 12\% of variance explained; fig. 3; table 1, pt. B), while growth rate explained $32 \%$ of metapopulation size. Interestingly, both the dispersal strategies of genotypes and environmental variability regimes affected metapopulation size, but the interactions between environmental variability and dispersal-related traits appeared nonsignificant (table 1, pt. B).

In contrast to metapopulation size, we found significant interactions between environmental variability regime and dispersal strategies on spatial variability in population size (fig. 3; table 1, pt. B). When resources varied spatially (i.e., spatial and spatiotemporal variability regimes), an increase in dispersal specialization and resource dependency (PC1) was associated with a reduced spatial variability of population size (fig. 3). We also found a significant interaction between environmental variability and density-dependent dispersal (PC2) on population spatial variability (table 1, pt. B): positive density-dependent dispersal was associated with amplified effects of spatiotemporal environmental variability for population spatial variability, while negative densitydependent dispersal was associated with reduced population spatial variability.

Finally, temporal variability in metapopulation size appeared to be mostly driven by genotype growth rate, with $26 \%$ of total variance attributed to growth rate and $39 \%$ to environmental variability regimes (table 1, pt. B).

\section{Discussion}

Intraspecific variability can be considerable, often equaling or even exceeding interspecific variability (Des Roches et al. 2018; Raffard et al. 2019). Since phenotypic traits not only are the target of selection but can influence ecological dynamics (Hendry 2017), assessing whether and to what extent phenotypic variability matters for ecological and evolutionary dynamics compared with environmental variability is crucial (Des Roches et al. 2018; Raffard et al. 2019). Here we experimentally demonstrated for the first time that differences among genotypes within a species may be at least as important as spatial and temporal variability in habitat quality for metapopulation dynamics in simple two-patch microcosms. Furthermore, we showed that a great proportion of this intraspecific variability effect results from differences among genotypes in dispersal syndromes.

\section{Architecture of Dispersal Syndromes}

Dispersal-related traits organize into two strategies in Tetrahymena thermophila. A first dimension reflects dispersal 

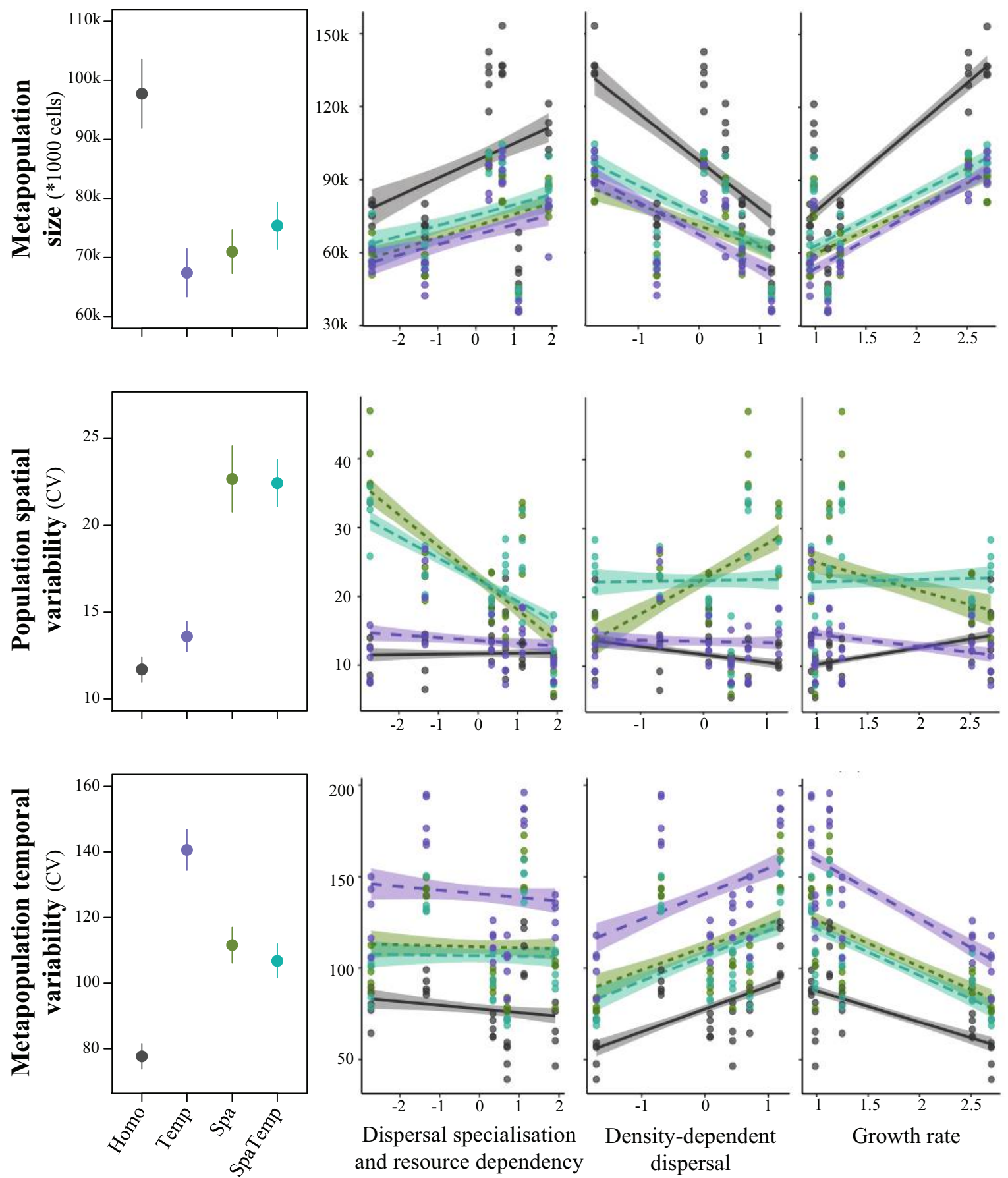

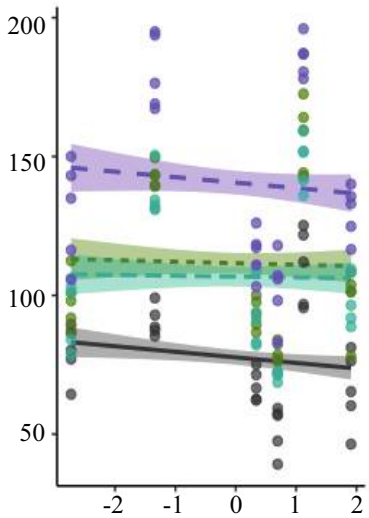

Dispersal specialisation and resource dependency

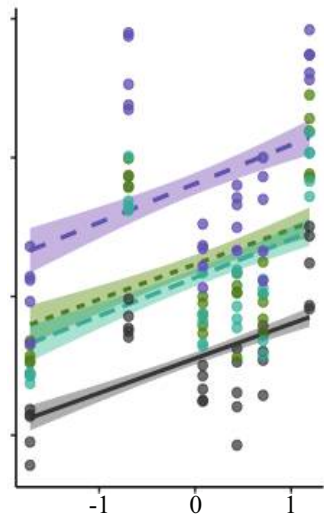

Density-dependent dispersal

Figure 3: Dispersal syndromes and environmental variability affect metapopulation dynamics. Colors correspond to environmental variability regimes. Left panels show the effects of environmental variability regimes on average values of the dynamics of two-patch microcosms (i.e., all genotypes; mean \pm SE). In right panels, lines show predicted values from linear regressions of metapopulation variables in the function of genotypes' dispersal strategies and growth rate. Colored areas represent the $50 \%$ confidence interval of the regressions. CV $=$ coefficient of variation. 
Table 1: Statistics of the drivers of the dynamics of simple experimental metapopulations

\begin{tabular}{|c|c|c|c|c|c|c|c|c|c|}
\hline & \multicolumn{3}{|c|}{ Metapopulation size } & \multicolumn{3}{|c|}{ Spatial variability } & \multicolumn{3}{|c|}{ Temporal variability } \\
\hline & $F(\mathrm{df})$ & $P$ & $R^{2}$ & $F(\mathrm{df})$ & $P$ & $R^{2}$ & $F(\mathrm{df})$ & $P$ & $R^{2}$ \\
\hline \multicolumn{10}{|l|}{ Part A: } \\
\hline Genotype & $152.63(5,96)$ & $<.001$ & .73 & $3.28(5,96)$ & .009 & .24 & $20.68(5,96)$ & $<.001$ & .52 \\
\hline $\begin{array}{l}\text { Environmental variability } \\
\text { regime }\end{array}$ & $11.99(3,96)$ & $<.001$ & .19 & $43.94(3,96)$ & $<.001$ & .34 & $21.87(3,96)$ & $<.001$ & .39 \\
\hline $\begin{array}{l}\text { Genotype } \times \text { environmental } \\
\text { variability }\end{array}$ & $5.90(15,96)$ & $<.001$ & .04 & $6.65(15,96)$ & $<.001$ & .22 & $2.77(15,96)$ & .001 & .03 \\
\hline Part B: & & & & & & & & & \\
\hline $\begin{array}{l}\text { PC1: dispersal specialization } \\
\text { and resource dependency }\end{array}$ & $14.47(1,113)$ & $<.001$ & .06 & $.05(1,104)$ & .817 & .13 & & & \\
\hline $\begin{array}{l}\text { PC2: density-dependent } \\
\text { dispersal }\end{array}$ & $6.65(1,113)$ & .011 & .12 & $.01(1,104)$ & .948 & .01 & & & \\
\hline Growth rate & $70.57(1,113)$ & $<.001$ & .32 & $2.75(1,104)$ & .114 & .01 & $84.00(1,115)$ & $<.001$ & .26 \\
\hline $\begin{array}{l}\text { Environmental variability } \\
\text { regime }\end{array}$ & $24.22(3,113)$ & $<.001$ & .19 & $4.13(3,104)$ & .008 & .34 & $41.69(3,115)$ & $<.001$ & .39 \\
\hline Environmental variability $\times \mathrm{PC} 1$ & & & & $16.13(3,104)$ & $<.001$ & .12 & & & \\
\hline Environmental variability $\times$ PC2 & & & & $8.27(3,104)$ & $<.001$ & .07 & & & \\
\hline $\begin{array}{l}\text { Environmental variability } \times \\
\text { growth rate }\end{array}$ & & & & $2.83(3,104)$ & .042 & .03 & & & \\
\hline
\end{tabular}

Note: Part A shows the quantification of the importance of genotype (intraspecific variability), environmental variability regime, and their interaction on metapopulation dynamics descriptors. Part B shows the contribution of dispersal syndromes, growth rate, and environmental variability regimes to variability in the dynamics of two-patch microcosms. Nonsignificant interactions were removed from the models following a backward-selection procedure. PC $=$ principal component.

specialization comprising multiple correlated traits (i.e., dispersal propensity, phenotypic specialization for dispersal, and the ability to preferentially leave poor patches compared with rich ones), in agreement with previous studies that defined dispersal specialization as the combination of multiple traits organized in a syndrome, beyond simply an increased tendency to disperse (Clobert et al. 2009; Stevens et al. 2014; Cote et al. 2017). The second dimension includes densitydependent dispersal, a component of dispersal strategies that appears to covary with growth rate (fig. $2 a$ ). High growth rate is associated with a tendency to decrease dispersal rate when density increases (i.e., they preferentially stay in more crowded patches), while low-growth-rate genotypes show positive density-dependent dispersal, preferentially leaving high-density patches. This suggests that high-growth-rate genotypes might use density as a cue of habitat quality, as suggested in previous studies (McPeek and Holt 1992; Poethke et al. 2011; Pennekamp et al. 2014; Jacob et al. 2016b), preferentially staying in large and dense populations. Furthermore, we found that growth rate was decoupled from dispersal specialization (fig. 2a). Although dispersal has been repeatedly found to correlate with traits linked to reproduction (e.g., Duckworth and Badyaev 2007; Stevens et al. 2014; Bonte and Dahirel 2017; reviewed in Ronce and Clobert 2012), the way dispersal-related traits covary and the form of the resulting dispersal syndromes can be shaped by the environmental context and may thus differ among populations and species (Legrand et al. 2016; Cote et al. 2017). This points out the importance of investigating the occurrence and drivers of variability in dispersal syndromes for our understanding of dispersal evolution.

\section{Genotype-Dependent Metapopulation Dynamics}

As expected since resources affect growth rate, the environmental variability treatments consisting of spatial and spatiotemporal variability of resources increased spatial variability in population size (fig. 3). Beyond these environmental variability effects on metapopulation dynamics, a major finding of this experimental study is that variability among genotypes may explain as much variation in the dynamics of experimental two-patch microcosms as environmental variability (fig. 4a). Furthermore, these differences among genotypes interact with environmental variability in driving these dynamics (fig. 4a). While environmental variability is probably the most intuitive driver of metapopulation dynamics, our results provide experimental evidence for a potential great importance of intraspecific variability for ecological dynamics. Furthermore, the effects of environmental variability for metapopulation dynamics differ among genotypes. The large intraspecific variability found in numerous organisms (Albert et al. 2010; Violle et al. 2012) may therefore also be an important factor driving metapopulation dynamics (Bolnick et al. 2003; Cote et al. 2017). 


\section{a) Genotype}

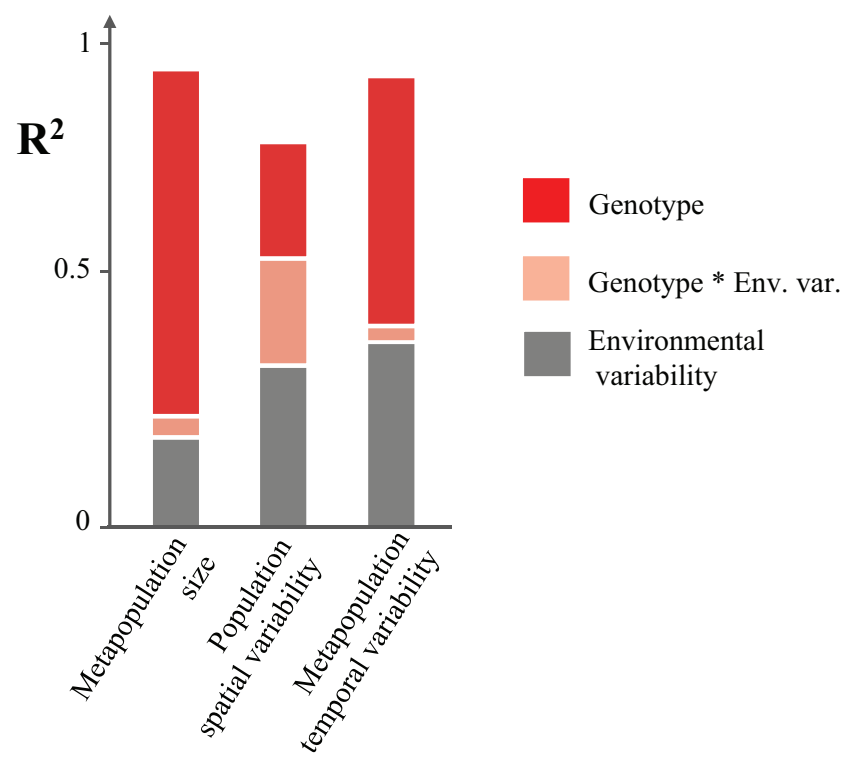

\section{b) Dispersal syndromes}

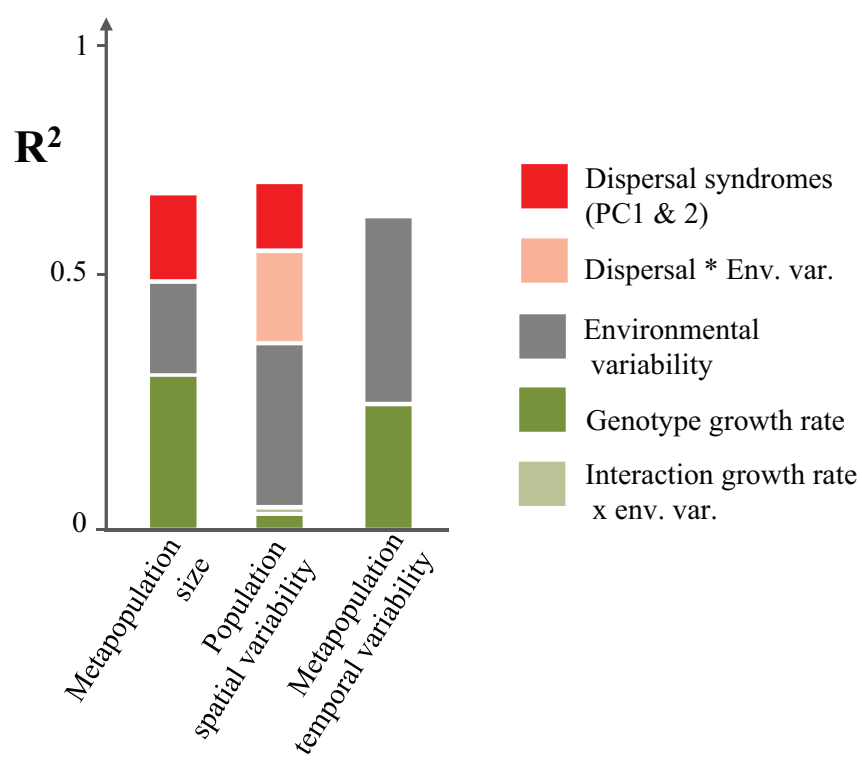

Figure 4: Relative contribution of the drivers of the dynamics of simple experimental metapopulations. $a$, Contribution of intraspecific variability (i.e., genotype), environmental variability regime, and their interaction on metapopulation dynamics descriptors. $b$, Contribution of dispersal syndromes, growth rate, and environmental variability regimes to variability in the dynamics of two-patch microcosms. The size of colored bars is proportional to the $R^{2}$ of the different potential drivers. Associated statistics are provided in table 1 .

Homogeneous environments result in larger metapopulation sizes compared with spatially and/or temporally variable environments for all genotypes. Although our experiment was designed to ensure that all two-patch microcosms would contain an identical total quantity of resources over time, the relationship between resources and growth rate in single nonconnected tubes was nonlinear: in- creasing resources from low resources to half of standard significantly increased growth rate $\left(F_{1,34}=9.93, P=.003\right)$, but further increasing to standard resource concentration did not significantly increase growth rate $\left(F_{1,34}=0.005\right.$, $P=.95)$. Consequently, although all environmental variability treatments contained the same quantity of resources at the two-patch microcosm level, such a nonlinear relationship 
may lead to higher growth in homogeneous microcosms compared with other regimes and thus likely explains the higher metapopulation size found in homogeneous environments (fig. 2). However, since the effects of resource concentration on growth rate did not significantly differ among genotypes (see "Methods"), this nonlinear relationship would not affect our conclusions regarding genotype and genotype $x$ environmental variability interaction effects on metapopulation dynamics.

\section{Dispersal Syndromes and Environmental Variability Drive Metapopulation Dynamics}

The consequences of dispersal for spatially structured populations are predicted to differ depending on the dispersalrelated traits involved (Bowler and Benton 2005; Clobert et al. 2009; Jacob et al. 2015a). Here we found that variability in dispersal syndromes accounted for one-fifth to onethird of variability in the dynamics of experimental metapopulations (fig. 4b). As anticipated, given the importance of the dispersal process in metapopulation dynamics (Ronce 2007; Clobert et al. 2012), this study demonstrates that variability in dispersal syndromes may be responsible for more than one-third of the variability in simple experimental metapopulations. Interestingly, however, dispersal syndromes and growth rate explained a lower percentage of variance in metapopulation dynamics than genotypes did (fig. 4). This result highlights that other phenotypic traits not measured in this study might play a significant role in driving metapopulation dynamics, such as cooperation strategy (Schtickzelle et al. 2009; Chaine et al. 2010; Jacob et al. 2016b), information use abilities (Jacob et al. 2015b, 2017, 2018), resistance to starvation (Fjerdingstad et al. 2007), or ecological specialization (Jacob et al. 2018).

Increased dispersal movements are predicted to homogenize metapopulations, thus reducing spatial variability in population size (Briggs and Hoopes 2004; Vogwill et al. 2009; Abbott 2011; Wang et al. 2015). Accordingly, we found that increased dispersal propensity coupled with phenotypic specialization and the ability to preferentially leave poor patches reduces spatial variability in population size (fig. 3). Interestingly, dispersal generally incurs costs arising from increased movements toward poor habitats in sourcesink contexts or mortality rates during movement (Abbott 2011; Bonte et al. 2012). Consequently, we would expect increased dispersal propensity alone without phenotypic specialization or plasticity in dispersal decisions to have negative effects on metapopulation size. On the contrary, here we found that increasing dispersal specialization and resource dependency led to increased metapopulation size (fig. 2). This pattern might result from this coupling of phenotypic specialization for dispersal and context-dependent dispersal, which is expected to increase the success of move- ments between patches and thus improve metapopulation persistence (Edelaar et al. 2008; Clobert et al. 2009; Jacob et al. 2015a). Furthermore, we found that positive densitydependent dispersal was associated with decreased metapopulation size and increased spatial variability of population size under spatiotemporal environmental variability (fig. 3; table 1, pt. B). Theoretical work predicts negative density-dependent dispersal to be selected in spatially heterogeneous environments that are temporally stable, while spatiotemporal variability should favor positive densitydependent dispersal (Rodrigues and Johnstone 2014). Overall, these results highlight the importance of experimentally quantifying the consequences of different architectures of dispersal syndromes for metapopulation dynamics under different degrees and directions of environmental changes.

\section{Conclusion}

Dispersal is a core process driving population dynamics in spatially structured environments (Clobert et al. 2012). Improving our ability to predict the dynamics and persistence of populations facing environmental changes crucially requires accounting for the complexity of the dispersal process (Clobert et al. 2009; Travis et al. 2012; Cote et al. 2017). Considering not only mean values of phenotypic traits but variance and covariance between the multiple phenotypic and life-history traits related to dispersal is an important challenge (Laughlin and Messier 2015; Cote et al. 2017). Here we provide the first experimental evidence for the role played by intraspecific variability in dispersal syndromes in driving metapopulation dynamics. Variability in dispersal decisions, related phenotypic traits, and their organization in strategies might indeed profoundly modify how organisms respond to environmental changes. Investigating the conditions favoring the evolution of different dispersal syndromes, their coexistence, and the metapopulation consequences of a diversity of competing dispersal strategies thus represent a central future step. Intraspecific variability of dispersal syndromes furthermore appeared to be important for metapopulation dynamics as a central aspect of the environment that is resource availability in our simple experimental metapopulations.

To reach this conclusion, we used simple laboratory microcosms, that is, highly controlled and replicated experimental systems providing a unique opportunity to study the causality of tested factors (Jessup et al. 2004; Benton et al. 2007), especially in the context of dispersal (Haddad 2012). These simplified worlds often lack the necessary realism to fully explain natural patterns (Srivastava et al. 2004), being by definition of extreme simplicity compared with the plethora of environmental factors that play in nature. The degree to which intraspecific variability influences metapopulation dynamics is consequently expected to change 
between our specific experimental situation and a natural system, the challenge being to know how much. Furthermore, here we separately quantified the effects of isolated genotypes and phenotypes for metapopulation dynamics, a scenario that contrasts with many natural populations that are genetically diversified (with the notable exception of habitats recently colonized by a few individuals). Future experiments are now required to determine how genetic diversity affects ecological dynamics and to identify the environmental conditions that are required to maintain local polymorphism in dispersal syndromes. Nonetheless, by highlighting the high importance of intraspecific variability in metapopulation dynamics in simple microcosms, our study should help build and calibrate complementary theoretical models (Cote et al. 2017; Jacob et al. 2018). It should also stimulate future research effort required to assess the degree of generality of our results in more complex environments and landscape configurations. Finally, the present experiment provides evidence that the integration of variability in dispersal syndromes would improve models forecasting population and species response to environmental changes (Travis et al. 2013; Urban et al. 2013).

\section{Acknowledgments}

We thank Bart Haegeman for very helpful discussions and comments on the manuscript. This study was supported by Agence Nationale de la Recherche (ANR) INDHET (Individual Heterogeneity in Dispersal and Metapopulation Dynamics), ANR Netselect, and a MOVE-IN Louvain Marie Curie Action fellowship to S.J. S.J was funded by the Research Foundation-Flanders (FWO) research community EVENET (Eco-Evolutionary Network of Biotic Interactions) and a networking grant from Université catholique de Louvain (FEEDING). This work is part of the Laboratoire d'Excellence (LABEX) entitled TULIP (ANR-10-LABX-41).

Statement of authorship: S.J., A.S.C., J.C., and D.L. defined the research theme; S.J., A.S.C., M.H., J.C., and D.L. set up the experimental protocols; S.J., M.H., and D.L. performed the experiments; S.J. analyzed the data and wrote the manuscript; and all authors contributed substantially to revisions.

\section{Data and Code Availability}

The data supporting the findings of this study are available in the Dryad Digital Repository (https://doi.org/10.5061 /dryad.r63jf31; Jacob et al. 2019).

\section{Literature Cited}

Abbott, K. C. 2011. A dispersal-induced paradox: synchrony and stability in stochastic metapopulations. Ecology Letters 14:1158-1169.
Albert, C. H., W. Thuiller, N. G. Yoccoz, R. Douzet, S. Aubert, and S. Lavorel. 2010. A multi-trait approach reveals the structure and the relative importance of intra- vs. interspecific variability in plant traits: intra- vs. interspecific variability in plant traits. Functional Ecology 24:1192-1201.

Altermatt, F., E. A. Fronhofer, A. Garnier, A. Giometto, F. Hammes, J. Klecka, D. Legrand, et al. 2015. Big answers from small worlds: a user's guide for protist microcosms as a model system in ecology and evolution. Methods in Ecology and Evolution 6:218-231.

Bascompte, J., and V. Solé. 1998. Modeling spatiotemporal dynamics in ecology. Springer, Berlin.

Benton, T. G., M. Solan, J. M. J. Travis, and S. M. Sait. 2007. Microcosm experiments can inform global ecological problems. Trends in Ecology and Evolution 22:516-521.

Bertrand, F., and M. Maumy-Bertrand. 2010. Initiation à la Statistique avec R. Dunod, Paris.

Bolnick, D. I., P. Amarasekare, M. S. Araújo, R. Bürger, J. M. Levine, M. Novak, V. H. Rudolf, et al. 2011. Why intraspecific trait variation matters in community ecology. Trends in Ecology and Evolution 26:183-192.

Bolnick, D. I., R. Svanbäck, J. A. Fordyce, L. H. Yang, J. M. Davis, C. D. Hulsey, and M. L. Forister. 2003. The ecology of individuals: incidence and implications of individual specialization. American Naturalist 161:1-28.

Bonte, D., and M. Dahirel. 2017. Dispersal: a central and independent trait in life history. Oikos 126:472-479.

Bonte, D., H. Van Dyck, J. M. Bullock, A. Coulon, M. Delgado, M. Gibbs, V. Lehouck, et al. 2012. Costs of dispersal. Biological Reviews 87:290-312.

Bowler, D. E., and T. G. Benton. 2005. Causes and consequences of animal dispersal strategies: relating individual behaviour to spatial dynamics. Biological Reviews 80:205-225.

Briggs, C., and M. Hoopes. 2004. Stabilizing effects in spatial parasitoidhost and predator-prey models: a review. Theoretical Population Biology 65:299-315.

Chaine, A. S., N. Schtickzelle, T. Polard, M. Huet, and J. Clobert. 2010. Kin-based recognition and social aggregation in a ciliate. Evolution 54:1290-1300.

Clobert, J., M. Baguette, T. G. Benton, and J. Bullock. 2012. Dispersal ecology and evolution. Oxford University Press, Oxford.

Clobert, J., J.-F. Le Galliard, J. Cote, S. Meylan, and M. Massot. 2009. Informed dispersal, heterogeneity in animal dispersal syndromes and the dynamics of spatially structured populations. Ecology Letters 12:197-209.

Cote, J., E. Bestion, S. Jacob, J. Travis, D. Legrand, and M. Baguette. 2017. Evolution of dispersal strategies and dispersal syndromes in fragmented landscapes. Ecography 40:56-73.

De Roissart, A., S. Wang, and D. Bonte. 2015. Spatial and spatiotemporal variation in metapopulation structure affects population dynamics in a passively dispersing arthropod. Iournal of Animal Ecology 84:1565-1574.

Des Roches, S., D. M. Post, N. E. Turley, J. K. Bailey, A. P. Hendry, M. T. Kinnison, J. A. Schweitzer, et al. 2018. The ecological importance of intraspecific variation. Nature Ecology and Evolution 2:57-64.

Duckworth, R. A., and A. V. Badyaev. 2007. Coupling of dispersal and aggression facilitates the rapid range expansion of a passerine bird. Proceedings of the National Academv of Sciences of the USA 104:15017-15022.

Duputié, A., and F. Massol. 2013. An empiricist's guide to theoretical predictions on the evolution of dispersal. Interface Focus 3:20130028. 
Edelaar, P., A. M. Siepielski, and J. Clobert. 2008. Matching habitat choice causes directed gene flow: a neglected dimension in evolution and ecology. Evolution 62:2462-2472.

Endler, J. 1980. Natural selection on color patterns in Poecilia reticulata. Evolution 34:76-91.

Fjerdingstad, E. J., N. Schtickzelle, P. Manhes, A. Gutierrez, and J. Clobert. 2007. Evolution of dispersal and life history strategiesTetrahymena ciliates. BMC Evolutionary Biology 7:133.

Fronhofer, E. A., and F. Altermatt. 2015. Eco-evolutionary feedbacks during experimental range expansions. Nature Communications 6:6844.

Gause, G. 1934. The struggle for existence. Dover, Mineaola, NY.

Gibert, J. 2016. The effect of phenotypic variation on metapopulation persistence. Population Ecology 58:345-355.

Groemping, U. 2006. Relative importance for linear regression in R: the package relaimpo. Journal of Statistical Software 17, doi:10.18637 /jss.v017.i01.

Haddad, N. 2012. Connecting ecology and conservation through experiment. Nature Methods 9:794-795.

Hanski, I. 1998. Metapopulation dynamics. Nature 396:41-49.

Hanski, I., and O. Gaggiotti. 2004. Ecology, genetics and evolution of metapopulations. Elsevier, Amsterdam.

Hendry, A. P. 2017. Eco-evolutionary dynamics. Princeton University Press, Princeton, NJ.

Holt, R., and M. Barfield. 2015. The influence of imperfect matching habitat choice on evolution in source-sink environments. Evolutionary Ecology 29:887-904.

Jacob, S., E. Bestion, D. Legrand, J. Clobert, and J. Cote. 2015a. Habitat matching and spatial heterogeneity of phenotypes: implications for metapopulation and metacommunity functioning. Evolutionarv Ecology 29:851-871.

Jacob, S., A. S. Chaine, M. Huet, J. Clobert, and D. Legrand. 2019. Data from: Variability in dispersal syndromes is a key driver of metapopulation dynamics in experimental microcosms. American Naturalist, Dryad Digital Repository, https://doi.org/10.5061/dryad .r63jf31.

Jacob, S., A. S. Chaine, N. Schtickzelle, M. Huet, and J. Clobert. 2015b. Social information from immigrants: multiple immigrantbased sources of information for dispersal decisions in a ciliate. Journal of Animal Ecology 84:1373-1383.

Jacob, S., J. Clobert, D. Legrand, N. Schtickzelle, and A. Chaine. 2016a. Social information in cooperation and dispersal in Tetrahymena. Pages 235-252 in G. Witzany and M. Nowacki, eds. Biocommunication of ciliates. Springer, Basel.

Jacob, S., E. Laurent, B. Haegeman, R. Bertrand, J. Prunier, D. Legrand, J. Cote, et al. 2018. Habitat choice meets thermal specialization: competition with specialists may drive sub-optimal habitat preferences in generalists. Proceedings of the National Academv of Sciences of the USA 115:11988-11993.

Jacob, S., D. Legrand, A. Chaine, D. Bonte, N. Schtickzelle, M. Huet, and J. Clobert. 2017. Gene flow favours local adaptation under habitat choice in ciliate microcosms. Nature Ecology and Evolution 1:1407-1410.

Jacob, S., P. Wehi, J. Clobert, D. Legrand, N. Schtickzelle, M. Huet, and A. Chaine. 2016b. Cooperation-mediated plasticity in dispersal and colonization. Evolution 70:2336-2345.

Jessup, C. M., R. Kassen, S. E. Forde, B. Kerr, A. Buckling, P. B. Rainey, and B. J. M. Bohannan. 2004. Big questions, small worlds: microbial model systems in ecology. Trends in Ecology and Evolution 19:189-197.
Kettlewell, H. 1973. The evolution of melanism. Oxford University Press, Oxford.

Laughlin, D. C., and J. Messier. 2015. Fitness of multidimensional phenotypes in dynamic adaptive landscapes. Trends in Ecology and Evolution 30:487-496.

Legrand, D., J. Cote, E. A. Fronhofer, R. D. Holt, O. Ronce, N. Schtickzelle, J. M. J. Travis, et al. 2017. Eco-evolutionary dynamics in fragmented landscapes. Ecography 40:9-25.

Legrand, D., O. Guillaume, M. Baguette, J. Cote, A. Trochet, O. Calvez, S. Zajitschek, et al. 2012. The Metatron: an experimental system to study dispersal and metaecosystems for terrestrial organisms. Nature Methods 9:828-833.

Legrand, D., N. Larranaga, R. Bertrand, S. Ducatez, O. Calvez, V. M. Stevens, and M. Baguette. 2016. Evolution of a butterfly dispersal syndrome. Proceedings of the Roval Society B 283:20161533.

Legrand, D., A. Trochet, S. Moulherat, O. Calvez, V. M. Stevens, S. Ducatez, J. Clobert, et al. 2015. Ranking the ecological causes of dispersal in a butterfly. Ecography 38:822-831.

Leimar, O., and U. Norberg. 1997. Metapopulation extinction and genetic variation in dispersal-related traits. Oikos 80:448.

Lenormand, T. 2002. Gene flow and the limits to natural selection. Trends in Ecology and Evolution 17:183-189.

Levin, S. A. 1976. Population dynamic models in heterogeneous environments. Annual Review of Ecology and Systematics 7:287-310.

Lindeman, R., P. Merenda, and R. Gold. 1980. Introduction to bivariate and multivariate analysis. Scott, Foresman, Glenview, IL.

MacArthur, R., and E. Wilson. 1963. An equilibrium theory of insular zoogeography. Evolution 17:373-387.

McPeek, M., and R. Holt. 1992. The evolution of dispersal in spatially and temporally varying environments. American Naturalist 140:10101027.

Moran, P. 1953. The statistical analysis of the Canadian lynx cycle. II. Synchronization and meteorology. Australian Journal of Zoology 1:281-298.

Mortier, F., S. Jacob, M. Vandegehuchte, and D. Bonte. 2019. Habitat choice stabilizes metapopulation dynamics by enabling ecological specialisation. Oikos 128:529-539

Nelsen, E. M. 1978. Transformation in Tetrahymena thermophila: development of an inducible phenotype. Developmental Biology 66:17-31.

Pelletier, F., D. Garant, and A. P. Hendry. 2009. Eco-evolutionary dynamics. Philosophical Transactions of the Roval Societv B 364:14831489.

Pennekamp, F., K. A. Mitchell, A. Chaine, and N. Schtickzelle. 2014. Dispersal propensity in Tetrahymena thermophila ciliates - a reaction norm perspective. Evolution 68:2319-2330.

Pennekamp, F., and N. Schtickzelle. 2013. Implementing image analysis in laboratory-based experimental systems for ecology and evolution: a hands-on guide. Methods in Ecology and Evolution 4:483-492.

Pimentel, D. 1968. Population regulation and genetic feedback. Science 159:1432-1437.

Poethke, H., C. Dytham, and T. Hovestadt. 2011. A Metapopulation paradox: partial improvement of habitat may reduce metapopulation persistence. American Naturalist 177:792-799.

Raffard, A., F. Santoul, J. Cucherousset, and S. Blanchet. 2019. The community and ecosystem consequences of intraspecific diversity: a meta-analysis. Biological Reviews 94:648-661.

Rodrigues, A. M. M., and R. A. Johnstone. 2014. Evolution of positive and negative density-dependent dispersal. Proceedings of the Roval Society B 281:20141226. 
Ronce, O. 2007. How does it feel to be like a rolling stone? ten questions about dispersal evolution. Annual Review of Ecology, Evolution and Systematics 38:231-253.

Ronce, O., and J. Clobert. 2012. Dispersal syndromes. Pages 119-138 in J. Clobert, M. Baguette, T. G. Benton, and J. M. Bullock, eds. Dispersal ecology and evolution. Oxford University Press, Oxford.

Schtickzelle, N., E. J. Fjerdingstad, A. Chaine, and J. Clobert. 2009. Cooperative social clusters are not destroyed by dispersal in a ciliate. BMC Evolutionary Biology 9:251.

Srivastava, D. S., J. Kolasa, J. Bengtsson, A. Gonzalez, S. P. Lawler, T. E. Miller, P. Munguia, et al. 2004. Are natural microcosms useful model systems for ecology? Trends in Ecology and Evolution 19:379384.

Stearns, S. 1983. The genetic basis of differences in life- history traits among six populations of mosquitofish (Gambusia affinis) that shared ancestors in 1905. Evolution 37:618-627.

Stevens, V. M., S. Whitmee, J.-F. Le Galliard, J. Clobert, K. BöhningGaese, D. Bonte, M. Brändle, et al. 2014. A comparative analysis of dispersal syndromes in terrestrial and semi-terrestrial animals. Ecology Letters 17:1039-1052.

Thompson, J. 1998. Rapid evolution as an ecological process. Trends in Ecology and Evolution 13:329-332.

Travis, J., M. Delgado, G. Bocedi, M. Baguette, K. Bartoń, D. Bonte, I. Boulangeat, et al. 2013. Dispersal and species' responses to climate change. Oikos 122:1532-1540.
Travis, J., K. Mustin, K. A. Bartoń, T. G. Benton, J. Clobert, M. M. Delgado, C. Dytham, et al. 2012. Modelling dispersal: an ecoevolutionary framework incorporating emigration, movement, settlement behaviour and the multiple costs involved: modelling dispersal. Methods in Ecology and Evolution 3:628-641.

Urban, M., M. Leibold, P. Amarasekare, L. Demeester, R. Gomulkiewicz, M. Hochberg, C. Klausmeier, et al. 2008. The evolutionary ecology of metacommunities. Trends in Ecology and Evolution 23:311-317.

Urban, M., P. Zarnetske, and D. Skelly. 2013. Moving forward: dispersal and species interactions determine biotic responses to climate change. Annals of the New York Academy of Science 1297:44-60.

Violle, C., B. J. Enquist, B. J. McGill, L. Jiang, C. H. Albert, C. Hulshof, V. Jung, et al. 2012. The return of the variance: intraspecific variability in community ecology. Trends in Ecology and Evolution 27:244-252.

Vogwill, T., A. Fenton, and M. A. Brockhurst. 2009. Dispersal and natural enemies interact to drive spatial synchrony and decrease stability in patchy populations. Ecology Letters 12:1194-1200.

Wang, S., B. Haegeman, and M. Loreau. 2015. Dispersal and metapopulation stability. Peer 3:e1295.

Associate Editor: Tom E. X. Miller Editor: Russell Bonduriansky

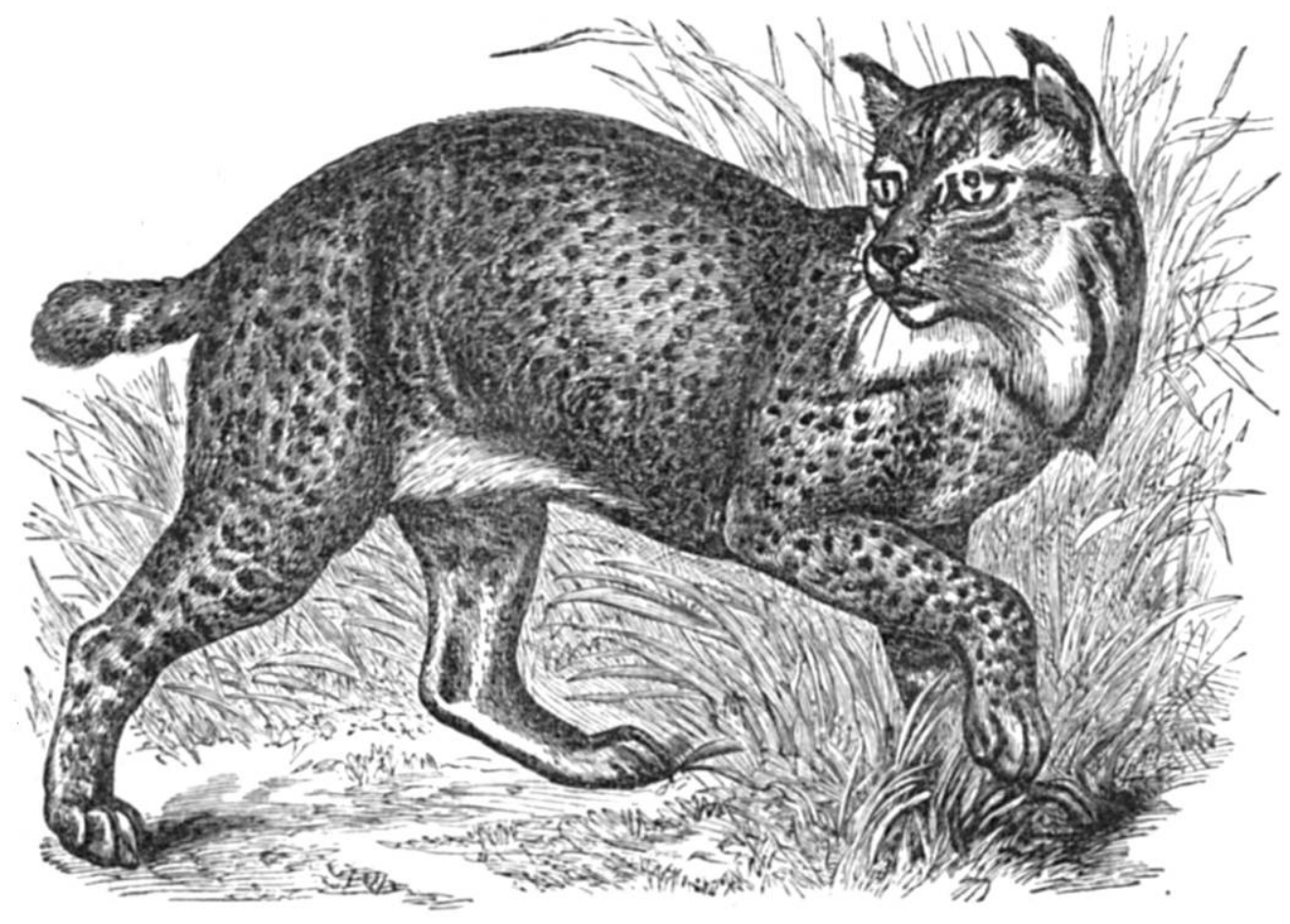

“The Northern Lynx, var. Felis maculata." From the review of Mivart's The Cat (The American Naturalist, 1882, 16:35-39). 